\title{
Autoregressive Cascades on Random Networks
}

\author{
Srikanth K. Iyer1 \\ Department of Mathematics, Indian Institute of Science, Bangalore, India. \\ Rahul Vaze 2 \\ School of Technology and Computer Science, Tata Institute of Fundamental Research, Homi Bhabha Road, \\ Mumbai, India. \\ Dheeraj Narsimha 3 \\ Birla Institute of Technology and Science (Goa) \\ Goa, India.
}

\begin{abstract}
This paper considers a model for cascades on random networks in which the cascade propagation at any node depends on the load at the failed neighbor, the degree of the neighbor as well as the load at that node. Each node in the network bears an initial load that is below the capacity of the node. The trigger for the cascade emanates at a single node or a small fraction of the nodes from some external shock. Upon failure, the load at the failed node gets divided randomly and added to the existing load at those neighboring nodes that have not yet failed. Subsequently, a neighboring node fails if its accumulated load exceeds its capacity. The failed node then plays no further part in the process. The cascade process stops as soon as the accumulated load at all nodes that have not yet failed is below their respective capacities. The model is shown to operate in two regimes, one in which the cascade terminates with only a finite number of node failures. In the other regime there is a positive probability that the cascade continues indefinitely. Bounds are obtained on the critical parameter where the phase transition occurs.
\end{abstract}

March 2, 2022

Keywords: Random graphs, trees, cascade, stability, networks.

\section{INTRODUCTION}

A model for the evolution of cascades in random networks was introduced in [1], to study the spread of ideas, opinion, technology etc. In this model, the nodes of a network are regarded as agents and the interactions between agents are modeled as links in the network. An agent initially in state 0 will adopt a new idea (state 1) as soon as a fraction of its neighbors who have adopted the new idea exceeds a threshold. The existence of a phase transition (as a function of the threshold) was demonstrated and a condition for the existence of global

1 skiyer@math.iisc.ernet.in, research supported in part by UGC center for advanced studies.

2 vaze@tcs.tifr.res.in, research supported in part by INSA young scientist award grant.

3 dheeraj.narasimha@gmail.com. cascades was derived. This model was generalized in [2], where in addition to the above, the links are endowed with random weights, whose distribution may depend on the degree of the two nodes that the link connects. The motivation for this model comes from the study of systemic risks in financial networks. A node adopts a new idea provided the sum of the weights of links to neighboring nodes that have adopted the idea exceeds a threshold that depends on the degree at that node. A further generalization is the threshold model [3, 4], where an agent adopts the new idea if the number of its neighbors or the combined edge weights of the neighbors that have adopted the new idea is larger than a random threshold.

The model that we study in this paper is motivated by networks such as the electrical networks or other networks where the nodes can be thought of as service providers and load sharing occurs via the net- 
work structure. Consider a large random network, where each node has degree that is distributed according to a specified distribution $\left\{w_{k}, k=0,1, \ldots\right\}$ (see [5]). Such a network is locally "tree-like" when the network size is large, that is, there are few short cycles. Melnik et. al. [6] examine the effectiveness of tree-like networks to study networks with clustering.

Initially, node $u$ bears a load $L_{u}^{(0)}$, and has capacity $c>0$, where the random variables $L_{u}^{(0)}$ are independent and whose distributions are the same as those of the non-negative random variable $L$. To begin with, let us assume that the support of $L$ is contained in $[0, c)$ (abbreviated as $L<c$ ). This restriction ensures that initially all nodes are stable or active. At time $t=0$, an external event or a surge occurs at a node that we label $r$, resulting in the load $L_{r}$ at that node to exceed $c$. This causes node $r$ to fail. If the number of neighbors of $r, N(r)=0$, then the cascade stops. If $N(r)=k>0$, then suppose that $u_{1}, \ldots, u_{k}$ are neighbors of $r$ with loads $L_{u_{1}}^{(0)}, \ldots L_{u_{k}}^{(0)}$, respectively at time $t=0$. Then at time $t=1$, a random fraction of the total load of the failed node $r$ is pushed over to each of the active neighbors, that is, the load at $u_{i}$ increases to

$$
L_{u_{i}}^{(1)}=p_{r u_{i}} L_{r}+L_{u_{i}}^{(0)}
$$

where $\left\{p_{r u_{i}} \geq 0, i=1, \ldots, k\right\}, \sum_{i=1}^{k} p_{r u_{i}}=1$, is random probability mass function. Consequently, a node whose new load exceeds its capacity, fails. Each failed node distributes its entire load among all its active (non-failed) neighbors randomly as described above. The cascade of failed nodes terminates if at some time, the resulting loads at all nodes that are neighbors of nodes that failed in the previous time step do not exceed their respective capacities. We will refer to this model as the autoregressive cascade (ARC) model or process, since the increase in load at a node as a result of failure of a neighboring node resembles an autoregressive process. We show the existence of a phase transition, that is, existence of regions where the cascade dies out in finite time with probability one, and regions where the process survives indefinitely with positive probability for infinite networks (or has a giant component of failed nodes for large finite networks).

ARC model is well-suited for studying the behavior of outages in electrical power networks, where typically a single node failure can lead to catastrophically wide-spread outages [7]. The ARC model is linked to the node based failure model in electrical networks, where nodes fail when the demand (load) overshoots the supply (capacity), and demand at the failed node is transferred to its active neighbors. In 7], simulation analysis is presented for a fully connected network with exponentially distributed loads. Simpler node failure models have been studied in 8, 9]. In particular, in 9], with each node failure, the load at every other active node is uniformly increased by a constant load, while in [8] each failing node results in the failure of a random number of nodes sampled at random from a given distribution. Both these models ignore the topology of the graph.

There is also a line failure model used in electrical networks [10, 11], where transmission lines fail when either the current or voltage exceeds line's rating, and failure of one transmission line changes the current/voltages change on other lines and failures propagate accordingly. ARC model does not capture the line failure model.

Note that the ARC model described above is different from the usual epidemic models (see for example [1]), where nodes fail or are infected based on some probabilistic or deterministic mechanism that depends only on the number of failed/infected neighbors but not the severity of the infection. These type of models are reviewed in [12, 13] and references therein. In contrast, in the ARC model, failure is governed by a mechanism of load transfer whose effect can persist over several generations.

The closest interaction model to the ARC model is the sandpile model [14 [16], where at each time slot, a particle is added at a randomly chosen node. Each node has a fixed capacity, and if the number of particles at any node exceeds its capacity, the node is said to topple and all the particles at that node are transferred equally to all its neighbors. Thus, the newly added particles at a node due to the toppling of a neighboring node is a fixed deterministic quantity, and given that a node has toppled, the future toppling of its neighbors are independent. With the ARC model, however, the transferred load is a random quantity, that correlates subsequent node failures. In case of the sandpile model, a toppled node is allowed to participate in further interactions, while in ARC model once a node fails it takes no further part in the process. There is also an inhibition sandpile model [17], where a toppled node is stopped temporarily or permanently from taking part in further interaction, however, only empirical results are available for the same. Another similar form of interaction is the bootstrap percolation, where the failure of a node depends on the failure of a fixed number of neighbors and not the weights at the failed neighboring nodes [18]. 
In this paper, we derive sufficient conditions for the cascade in the ARC process to survive indefinitely with positive probability or terminate with probability one in finite number of steps. We couple the ARC process with a suitable Galton-Watson branching process that lower bounds the growth of failed nodes in the ARC process. The condition for survival then follows from the condition for super-criticality of the coupled Galton-Watson process. For the converse result, we couple the ARC process with a suitable branching random walk (BRW) where particles are killed upon breaching a fixed barrier. The bound then follows from a result in [19] on the finite time termination of the BRW.

\section{ARC PROCESS}

We first study the evolution of the ARC process on an infinite graph $G$ with specified degree distribution $\left\{w_{k}, k \geq 0\right\}$. Although random graphs are only an abstraction, they have been widely used as first approximations [1]. For a random graph on $n$ vertices with specified degree distribution, the graph is locally "tree-like" in that there are no short cycles for large $n$. This becomes exact for the infinite graph.

If we start from a single failed node, then the number of neighbors of this node has distribution $\left\{w_{k}\right\}$. As we go forth, exploring the component starting from a single vertex or the progress of the cascade, we are interested in the unexplored nodes or nodes that have not yet failed. The first and subsequent generations will then have a size-biased degree distribution (see 20], Chapter 3, pp71). A first generation vertex with degree $k$ is $k$ times as likely to be chosen as the one with degree 1 . So the distribution of degree minus one of nodes in the first and subsequent generations is given by

$$
\tilde{w}_{k-1}=\frac{k w_{k}}{\mu}, \quad k \geq 1,
$$

where

$$
\mu=\sum_{k=1}^{\infty} k w_{k} .
$$

In (11), the subscript $k-1$ is used since we have used one edge in linking to that vertex. It is this sizebiased distribution $\tilde{w}_{k}$ given by (1) that is relevant as far as the long term behavior of the process is concerned.
Since we are interested in the survival or extinction of the ARC process, we require the underlying graph $G$ to have an infinite connected component. Thus, without loss of generality we will assume that $\tilde{\mu}=\sum_{k} k \tilde{w}_{k}>1$ (see Theorem 3.1.3, [20]) for which there is a positive probability that the component of $G$ containing any node is infinite. This condition also ensures that for a finite graph, there is a giant component containing a large fraction of the nodes with probability approaching one asymptotically in the size of the graph.

Each node $u \in G$ carries an initial load $L_{u}^{(0)}$ and has a fixed capacity $c$. The random variables $L_{u}^{(0)}$ are assumed to be independent and identically distributed. We denote by $L$ a generic random variable having the same distribution as the initial loads. Let $\mathrm{F}$ be the distribution function of $L$. Suppose that $\mathrm{F}$ is supported on $(0, c)$. Thus, to begin with, all nodes have loads less than their capacity. This condition is realistic for power networks where node failures are rare.

At time $t=0$, an external event happens resulting in the failure of the root node $r$, making $L_{r}^{(0)}>c$. If $r$ is isolated, then the cascade stops. Else, the load $L_{r}^{(0)}$ at $r$ is then transferred to its neighbors as described below. Given the degree $N(r)=k, k>0$, of the root node $r$, let $u_{1}, u_{2}, \ldots, u_{k}$ be the neighboring nodes of the root. Let $\left\{p_{r u_{i}}, i=1, \ldots, k\right\}$ be an exchangeable set of non-negative random variables satisfying $\sum_{i=1}^{k} p_{r u_{i}}=1$. That is, for any permutation $\sigma=(\sigma(1), \sigma(2), \ldots \sigma(k))$ of $\{1,2, \ldots, k\}$, we have

$\left(p_{r u_{1}}, p_{r u_{2}}, \ldots, p_{r u_{k}}\right) \stackrel{d}{=}\left(p_{r u_{\sigma(1)}}, p_{r u_{\sigma(2)}}, \ldots, p_{r u_{\sigma(k)}}\right)$.

In particular, the distribution of $p_{r u_{i}}$ is independent of $i$, and hence we let $p=p(k)$ to denote the random variable satisfying

$$
p(k) \stackrel{d}{=} p_{r u_{i}}(k),
$$

where $\stackrel{d}{=}$ denotes equality in distribution. We will often write $p_{u v}$ for $p_{u v}(k)$. For each of the $N(r)=k$ nodes, $u_{1}, \ldots, u_{k}$, attached to the root, the load $L_{u_{i}}^{(1)}$ at time $t=1$ becomes

$$
L_{u_{i}}^{(1)}=p_{r u_{i}} L_{r}^{(0)}+L_{u_{i}}^{(0)} .
$$

If $L_{u_{i}}^{(1)}<c, \forall i=1,2, \ldots, k$, then the cascade of node failures stops at time $t=1$. Otherwise, node $u_{i}$ fails at time 1 if the load $L_{u_{i}}^{(1)} \geq c$. If $r$ is the only node to which $u_{i}$ is connected, then the cascade goes 
no further along this path. Else, each neighbor $v_{j}$ of $u_{i}$ other than the root $r$ receives an additional load $p_{u_{i} v_{j}} L_{u_{i}}^{(1)}$, where conditional on the degree $N\left(u_{i}\right)+1$ of $u_{i}$, the collection $\left\{p_{u_{i} v_{j}}, j=1, \ldots, N\left(u_{i}\right)\right\}$ forms an independent exchangeable random probability mass function identical in distribution to the one above. The probability that one of the neighbors of $u_{j}$ is also neighbor of $r$ is much smaller than the probabilities of interest and can be ignored due to the locally tree-like nature of the random graph as is the standard practice in such analysis.

The process is said to terminate at time $T+1$ if some node at a distance $T$ from the root has failed and none of the nodes at distance $T+1$ fail at time $T+1$. Else the cascade continues. Our first result is a sufficient condition for the cascade to survive forever with positive probability. For two reals, $a, b$ let $a \vee b$ denote their maximum.

\section{SUPER-CRITICAL REGIME}

Theorem III.1 Consider the ARC process on the infinite random graph $G$ as described above. Then if

$$
\sum_{k=1}^{\infty} \tilde{w}_{k} k \mathbb{E}[\overline{\mathrm{F}}(c(1-p(k)))]>1,
$$

then the cascade survives indefinitely with positive probability, where $\overline{\mathrm{F}}=1-\mathrm{F}$ and $p(k)$ is as defined in (3).

Proof: The idea of the proof is to couple the ARC process with a super-critical Galton-Watson (GW) process as follows. Instead of transferring the actual load at a failed node to its neighbors/children, we will transfer an amount equal to the capacity $c$ which is less than the load at the failed node. To be specific, the GW process starts at time 0 with a single individual at the root. The $N(r)$ nodes $u_{1}, \ldots, u_{r}$ connected to the root are the possible children of the root in generation 1 . If $N(r)=0$, then the process terminates. If $N(r)>0$, then conditioned on $N(r)=k$, the random allocation probabilities $\left\{p_{r u_{i}}, i=1, \ldots, k\right\}$, and the loads $\left\{L_{u_{i}}^{(0)}, i=\right.$ $1, \ldots, k\}$, the node $u_{i}$ comes alive at time 1 in the GW process if the following condition is satisfied,

$$
p_{r u_{i}} c+L_{u_{i}}^{(0)} \geq c .
$$

Note that in comparison, the node $u_{i}$ fails in the
ARC process if

$$
L_{u_{i}}^{(1)}=p_{r u_{i}} L_{r}^{(0)}+L_{u_{i}}^{(0)} \geq c
$$

This procedure is repeated at each level to construct the GW process. In constructing the GW process, one can think of the net load $L_{u}$ of the parent that fails being replaced by its capacity $c$. Since at the time of failure, the load at node $u, L_{u}^{(1)}>c$, if a node comes alive in the GW process then it fails in the ARC process as well. Using the capacity and not the actual load of the failed node to determine the number of children in the GW process, not only gives a lower bound on the number of failed nodes, it also ensures that number of offspring are independent and identically distributed across generations $n \geq 1$ in the GW process.

Now we need to derive the condition for survival or super-criticality of this GW process. For any node $u$ that fails in any generation larger than one, let $N(u)+1$ be the degree of that node. $N(u)$ has the size-biased distribution $\left\{\tilde{w}_{k}, k \geq 0\right\}$. If $N(u)>0$, then conditional on $N(u)=k$, let $v_{1}, \ldots, v_{k}$, be the neighbors of $u$ in the next generation and let $p_{u v_{1}}, \ldots, p_{u v_{k}}$ be the random allocation. The probability that $v_{k}$ comes alive in the GW process is given by

$$
\begin{aligned}
q_{k} & =P\left[p_{u v_{i}} c+L_{v_{i}}^{(0)} \geq c \mid N(u)=k\right] \\
& =\mathbb{E}[\overline{\mathrm{F}}(c(1-p(k))]
\end{aligned}
$$

where $\overline{\mathrm{F}}=1-\mathrm{F}$ and $p(k)$ is as defined in (3). Thus, the expected number of offspring of $u$ in the GW process given $N(u)=k$ equals $k q_{k}$. Since $N(u)$ has distribution $\left\{\tilde{w}_{k}\right\}$, the GW process is super-critical as long as the mean number of offspring is greater than 1 which is true if condition (4) holds. If the GW process is super-critical, then the (XXX which) process survives indefinitely with positive probability (see Athreya and Ney, 1972, pp. 7). As noted above, if the GW process survives, then so does the ARC process.

The following corollary is immediate.

Corollary III.2 Suppose that the load allocation is uniform, that is, conditional on the degree at a failed node (other than the root) being $N+1$, each of the $N$ non-failed neighbor receives an equal fraction $p=\frac{1}{N}$ of the load of its failed parent, provided $N>0$. Then the condition (4) for the $A R C$ process to survive in- 
definitely with positive probability reduces to

$$
\sum_{k=1}^{\infty} \tilde{w}_{k} k \overline{\mathrm{F}}\left(\frac{c(k-1)}{k}\right)>1 .
$$

If in addition, the degree of each node is fixed, that is $N \equiv d>1$ is a constant, then (6) reduces to

$$
d\left(1-\mathrm{F}\left(\frac{c(d-1)}{d}\right)\right)>1 .
$$

The next two results are straightforward consequences of the coupling from below of the ARC process with a super-critical GW process as described in proof of Theorem III.1. In order to state the results we need some notation.

Suppose that the load allocation is uniform, that is, $p(k) \equiv 1 / k$. Given the random variable $N$ having the size-biased degree distribution $\left\{\tilde{w}_{k}, k \geq 0\right\}$, let $M=0$ if $N=0$ and given $N=j>0$, let $M$ be a binomial random variable with parameters $j$ and $q_{j}$, where

$$
q_{j}=\overline{\mathrm{F}}\left(\frac{c(j-1)}{j}\right) .
$$

Note that the $q_{j}$ defined in (5) reduces to the one defined in (8) when the load allocation is uniform. Define the probability mass function

$$
m_{k}=P[M=k]=\sum_{j=k}^{\infty}\left(\begin{array}{l}
j \\
k
\end{array}\right) q_{j}^{k}\left(1-q_{j}\right)^{j-k} \tilde{w}_{j},
$$

for $k=0,1, \ldots$ Let $\Phi(s)=\sum_{k=1}^{\infty} s^{k} m_{k}$, be the probability generating function of $\left\{m_{k}, k \geq 0\right\}$, and let $\eta$ be the smallest non-negative root of the equation $\Phi(s)=s$. With uniform allocation, the offspring distribution of the coupled branching process for the first and subsequent generations is same as that of $M$. Note that the left hand side expression in (6) is $\mathbb{E}[M]$. And $\gamma=\mathbb{E}[M]>1$ is the condition for the branching process to be super-critical. Let $W_{n}$ be the number of nodes that fail in the ARC process at time $n$, that is, these are nodes at distance $n$ from the root and fail.

Proposition III.3 Under condition (6), the ARC process survives indefinitely with probability exceeding $1-\eta$. If in addition we have $\sum_{k} k \log k m_{k}<\infty$, then on a set of probability at least $1-\eta$, we have

$$
\liminf _{n \rightarrow \infty} \frac{W_{n}}{\gamma^{n}}>0
$$

Proof: The results follow easily from the above coupling and the behavior of a super-critical branching process (see Athreya and Ney, 1972, pp. 7, 30).

The implication of this result is that when the cascade survives, it grows at an exponential rate. Thus, the cascade spreads rapidly if it does not die out quickly.

We now specialize the results for large finite graphs and examine the consequence of the branching process coupling. Let $G_{n}$ be a graph with $n$ vertices and degree distribution $\left\{w_{k}, k \geq 0\right\}$. Suppose that the load from a failed parent is distributed equally among all its non-failed neighbors, i.e. $p(k) \equiv 1 / k$. The number of first generation children of the coupled branching process has distribution

$$
\zeta_{k}=\sum_{j=k}^{\infty}\left(\begin{array}{l}
j \\
k
\end{array}\right) q_{j}^{k}\left(1-q_{j}\right)^{j-k} w_{j}, \quad k=0,1, \ldots,
$$

where $q_{j}$ is as defined in (8). Let $\Psi=\sum_{k=1}^{\infty} s^{k} \zeta_{k}$ be the probability generating function of $\left\{\zeta_{k}, k \geq 0\right\}$, and let $\eta$ be as defined above. The following result is now a consequence of Theorem 3.1.3 ([20]) and the branching process coupling described in the proof of Theorem II.1

Theorem III.4 A sufficient condition for the existence of a giant component in $G_{n}$ is that (6) holds. If this condition holds, then the fraction of vertices in the giant component exceeds $1-\Psi(\eta)$ asymptotically.

Remark III.5 If $\mathbb{P}(L>c)>0$, then for a graph with a large number of nodes, approximately a fraction $f=P[L>c]$ of nodes will be in a failed state to begin with. In the sub-critical regime, the cascades starting from these nodes will form small islands of failed nodes, where each island is of finite size. In the super-critical regime, however, multiple initial failed will lead to the formation of a unique giant component of failed nodes.

\section{SUB-CRITICAL REGIME}

We now give a sufficient condition for the cascade to last only a finite number of generations in the infinite random graph $G$ almost surely. Before we proceed, we need to describe a result of Biggins [19] on branching random walks (BRW). 
A BRW is a process that starts with a single individual labelled $r$ located at $x \in \mathbb{R}$. An individual labelled $u$ born at location $y \in \mathbb{R}$ lives for unit time at the end of which gives birth to offspring located according to the point process $y+Z_{u}$, where $Z_{u}$ is an independent copy of a point process $Z$. Let

$$
\begin{aligned}
\delta(\theta) & =\mathbb{E}\left[\int_{-\infty}^{\infty} e^{-\theta t} d Z(t)\right], \\
& =\mathbb{E}\left[\sum_{u} \exp \left(-\theta z_{u}\right)\right]
\end{aligned}
$$

where $\left\{z_{u}\right\}$ are the points of $Z$. Define the function

$$
\gamma(0)=\inf \{\delta(\theta): \theta \geq 0\} .
$$

The following result is a particular case of Theorem 2 of $[19]$.

Theorem IV.1 Let $Z^{(n)}(0)$ be the number of individuals of the $B R W$ located in the interval $(-\infty, 0]$. If $\gamma(0)<1$, then, almost surely, $Z^{(n)}(0)=0$ for all but finitely many $n$.

Note that the result is independent of the location of the initial individual. Let

$$
h:=\inf _{\theta \geq 0}\left\{\mathbb{E}\left[N e^{\theta(L-(1-p) c)}\right]\right\},
$$

where the random variable inside the expectation is taken to be zero if $N=0$ and given $N=k>0, p(k)$ is as defined in (3).

Theorem IV.2 Consider the ARC model on the graph $G$ as described above. If $h<1$, then the cascade starting at a node $r$ with any load $\ell>c$ will terminate in finite time with probability 1.

Proof: We will dominate the ARC process with a BRW process starting with a single individual located at $\ell$ to mimic the failing of the root with load $L_{r}^{(0)}=\ell>c$. To motivate the construction of the coupled BRW process, suppose that $(u, v)$ is an edge in the random graph $G$ and the ARC cascade upon reaching node $u$ at time $t$ results in the load at $u$ increasing to $L_{u}^{t} \geq c$. This will cause the node $u$ to fail and result in the load at node $v$ at time $t+1$ increasing to

$$
L_{v}^{(t+1)}=p_{u v} L_{u}^{(t)}+L_{v}^{(0)} .
$$

Hence the difference in the resultant loads at nodes $v$ and $u$, or the "drift" in the load satisfies

$L_{v}^{(t+1)}-L_{u}^{(t)}=-\left(1-p_{u v}\right) L_{u}^{(t)}+L_{v}^{(0)} \leq-\left(1-p_{u v}\right) c+L_{v}^{(0)}$,

where the last inequality follows since node $u$ fails at time $t$ and thus $L_{u}^{(1)} \geq c$. Hence

$L_{v}^{(t+1(}=L_{u}^{(t)}+\left(L_{v}^{(t+1)}-L_{u}^{(t)}\right) \leq L_{u}^{(t)}-\left(1-p_{u v}\right) c+L_{v}^{(0)}$.

The coupled BRW process is defined as follows. The process starts with a particle labelled $r$ located at $L_{r}^{(0)}=\ell>c$, which is the load at the time of failure of the root node. Suppose node $u$ fails at time $t$ in the ARC process. If the number of non-failed neighbors $N(u)$, of $u$ equals zero, then no child is born in the BRW process. Else given $N(u)=k$, then for each non-failed neighbor $v_{i}$ of $u$, an individual with the same label $v_{i}$ is born in the BRW process at time $t+1$. If the new load at node $v_{i}$ in the ARC process is

$$
L_{v_{i}}^{(t+1)}=p_{u v_{i}}(k) L_{u}^{(t)}+L_{v_{i}}^{(0)},
$$

then the location of $v_{i}$ in the BRW process will

$$
x_{v_{i}}^{(t+1)}=x_{u}^{(t)}-\left(1-p_{u v_{i}}(k)\right) c+L_{v}^{(0)},
$$

where $x_{u}^{(t)}$ is the location of the individual labelled $u$ in the BRW process. A barrier is kept at $c$ in the BRW process, that is if a child is born at location $x<c$, then it is killed.

Refer to Fig. 1, for an illustration of the coupling argument. In Fig. 1, suppose at time $t$, a node labelled $u$ fails in the ARC process, then a particle labelled $u$ is born in the BRW process located at $x_{u}^{(t)} \geq L_{u}^{(t)}$. Now, since node $u$ has failed, a fraction $p_{u v}$ of the load at node $u$ is transferred to node $v$, and if node $v$ fails as a consequence, then a child of the particle at $x_{u}^{(t)}$ is born in the BRW process at location $x_{v}^{(t+1)}$, where the displacement $x_{v}^{(t+1)}-x_{u}^{(t)}=L_{v}^{(0)}-\left(1-p_{u v}\right) c$. Thus, from (12), if node $v$ fails at time $t+1$ in the ARC process, that is, $L_{v}^{(t+1)} \geq c$, then a corresponding particle with label $v$ is born in the BRW process and has location $x_{v}^{(t+1)} \geq L_{v}^{(1)}$. Thus, the ARC process terminates if at any time there are no individuals in the BRW.

Let the coupled BRW process defined above be denoted by $X$. To apply Theorem IV.1 we consider 
AR Cascade

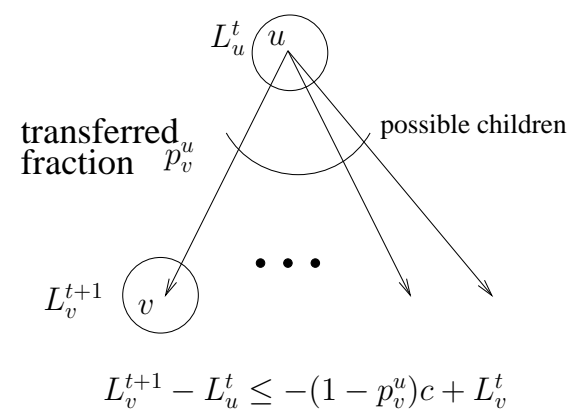

BRW process

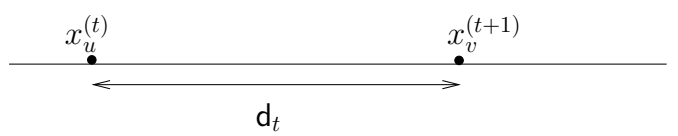

$\mathrm{d}_{t} \sim L-(1-p) c$

FIG. 1. Coupling the ARC model with BRW with a barrier.

another BRW $Y$ coupled to $X$ as follows. The $X, Y$ processes start off with one individuals located at $\ell,-\ell$ respectively. Starting with the initial ancestors, if a child is born to a parent located at $x$ in the BRW $X$ such that the location of the child is $x+d$, then a child is born to a corresponding parent located at $y=-x$ in the process $Y$ and the child is located at $y-d$. However, in the process $Y$, we do not kill individuals born at locations to the left of $c$, that is, there is no barrier. Thus in each generation, the individuals in $Y$ produce offspring whose numbers are distributed as $N$ with displacements distributed as $-(L-(1-p) c)$. Thus, the number of individuals in $Y$ in any generation is at least as large as in the process $X$. Moreover, note that if the BRW $Y$ drifts to the right, then the $X$ process drifts to the left.

Shift the origin to $-c$, so that the particle located at $x$ in process $X$ is at $x-c$ and particle located at $y$ in $Y$ process is at $-(y-c)$. Let $X_{n}([a, b])\left(Y_{n}([a, b])\right)$ be the number of individuals of process $X(Y)$ born at time $n$ and are located in the interval $[a, b]$. Then the BRW $X$ terminates if for some $n, X_{n}([2 c, \infty))=0$ and this happens if $Y_{n}((-\infty, 0]=0$. The location of the children of a node located at 0 in the process $Y$ is given by $Z=\left\{-\left(L_{i}-\left(1-p_{i}\right) c\right), i=1,2, \ldots, N\right\}$ and $N$ has distribution $\left\{\tilde{w}_{k}\right\}$. Thus for the process $Y$, we have

$$
\delta(\theta)=\mathbb{E}\left[N e^{\theta(L-(1-p) c)}\right],
$$

and hence $\gamma(0)=h$. Hence from Theorem IV.1 we have that for $h<1$, with probability one, the BRW $Y$ terminates in finite time. Hence the BRW $X$ terminates in finite time and consequently, so does the ARC process.

\section{SIMULATIONS}

In this section, to better understand the cascade dynamics we present some experimental results using Monte Carlo simulations, and compare our derived lower and upper bounds with the experimental thresholds at which cascade occurs. We consider both a random tree with a given degree distribution and a deterministic tree with fixed degree for each node. We let the tree to be of $N=10$ levels, and count the empirical measure of how often the cascade reaches any leaf at level $N$ as a measure of the number of failed nodes (probability of cascade) for large $N$ as a function of the capacity $c$.

In Figs. 2, 3, and 4, we plot the cascade probability for a deterministic tree with degree 2,3 , and 4 together with a random tree with Poisson distributed degree with mean 2,3 , and 4 , respectively. The load distribution is assumed to be a truncated Exponential distribution between 0.2 and 2 , respectively. The vertical lines on the left and right denote our derived lower and upper bounds (similar color) for each of the plots. We notice that the derived lower and upper bounds are fairly tight and give us a good estimate of the true threshold value. Also, as we increase the degree value, the derived bounds become tighter.

For a fixed tree (fixed number of children), the supercritical condition for cascade (7) can be rewritten as : $\mathrm{F}((d-1) c / d)<(d-1) / d$, where $d$ is the number of child nodes, $c$ is the capacity and F is the cumulative load distribution. If we consider the load distribution to be uniform between $[a b]$, and let $\frac{d-1}{d}=\beta$, then the super-critical condition for cascade is $\frac{(\beta c-a)}{(b-a)}<\beta$. Recall that $b<c$ (since we 
have assumed that $\mathrm{F}$ is supported on $(0, c))$. Then if $a=0$, condition $\frac{(\beta c-a)}{(b-a)}<\beta$ is never satisfied, so we pick $a$ greater than zero for all simulations. The same argument holds for the exponential load distribution case, and we simulate for truncated exponential load distribution between $[a, b]$ with $a>0$.

In Figs. 5] 6, and 7 , we plot the cascade probability for deterministic tree with degree 2, 3, and 4 for three different load distributions, exponential, power-law, and uniform, respectively. Once again, the vertical lines on the left and right denote our derived lower and upper bounds for cascade threshold for each of the plots. As before, we notice that the derived lower and upper bounds are fairly tight. Moreover, the bounds are tighter for exponential and power-law load distribution in comparison to uniform load distribution.

In Fig. 8, we plot the cascade probability for random tree with power-law degree distribution where degree $d$ is distributed as $P(d=k)=A k^{-\alpha}$ for uniform load distribution between [0.2 2] as a function of parameter $\alpha$. For reasonable simulation complexity, we renormalize the degree distribution to have at most 10 children, i.e. $k \leq 10$. We see that as the power-law exponent $\alpha$ increases, the derived bounds become loose. Finally, in Fig. 9, we plot the cascade probability for random tree with Poisson degree distribution for uniform load distribution between $[a, 2]$ as a function of parameter $a>0$. We see that as $a$ increases, i.e., as the load distribution becomes more concentrated, the derived bounds become loose.
[1] D. J. Watts, "A simple model of global cascades on random networks," Proceedings of the National Academy of Sciences, vol. 99, no. 9, pp. 5766-5771, 2002.

[2] J. P. Gleeson, T. Hurd, S. Melnik, and A. Hackett, "Systemic risk in banking networks without monte carlo simulation," in Advances in Network Analysis and its Applications. Springer, 2013, pp. 27-56.

[3] D. Kempe, J. Kleinberg, and É. Tardos, "Maximizing the spread of influence through a social network," in Proceedings of the ninth ACM SIGKDD international conference on Knowledge discovery and data mining. ACM, 2003, pp. 137-146.

[4] S. Venkatramanan and A. Kumar, "Information dissemination in socially aware networks under the linear threshold model," in Communications (NCC), 2011 National Conference on. IEEE, 2011, pp. 15.

[5] M. E. Newman, S. H. Strogatz, and D. J. Watts, "Random graphs with arbitrary degree distributions and their applications," Physical Review E, vol. 64, no. 2, p. 026118, 2001.

[6] S. Melnik, A. Hackett, M. A. Porter, P. J. Mucha, and J. P. Gleeson, "The unreasonable effectiveness of tree-based theory for networks with clustering,"
Physical Review E, vol. 83, no. 3, p. 036112, 2011.

[7] S. Kadloor and N. Santhi, "Understanding cascading failures in power grids," CoRR, vol. abs/1011.4098, 2010.

[8] I. Dobson, B. A. Carreras, and D. E. Newman, "A branching process approximation to cascading load-dependent system failure," in System Sciences, 2004. Proceedings of the 37th Annual Hawaii International Conference on. IEEE, 2004, pp. 10-pp.

[9] — , "A loading-dependent model of probabilistic cascading failure," Probability in the Engineering and Informational Sciences, vol. 19, no. 01, pp. 1532, 2005.

[10] A. Farina, A. Graziano, F. Mariani, and F. Zirilli, "Probabilistic analysis of failures in power transmission networks and phase transitions: study case of a high-voltage power transmission network," Journal of Optimization Theory and Applications, vol. 139, no. 1, pp. 171-199, 2008.

[11] H. Xiao and E. M. Yeh, "Cascading link failure in the power grid: A percolation-based analysis," in Communications Workshops (ICC), 2011 IEEE International Conference on. IEEE, 2011, pp. 1-6.

[12] M. Newman, Networks: an introduction. Oxford University Press, 2010. 


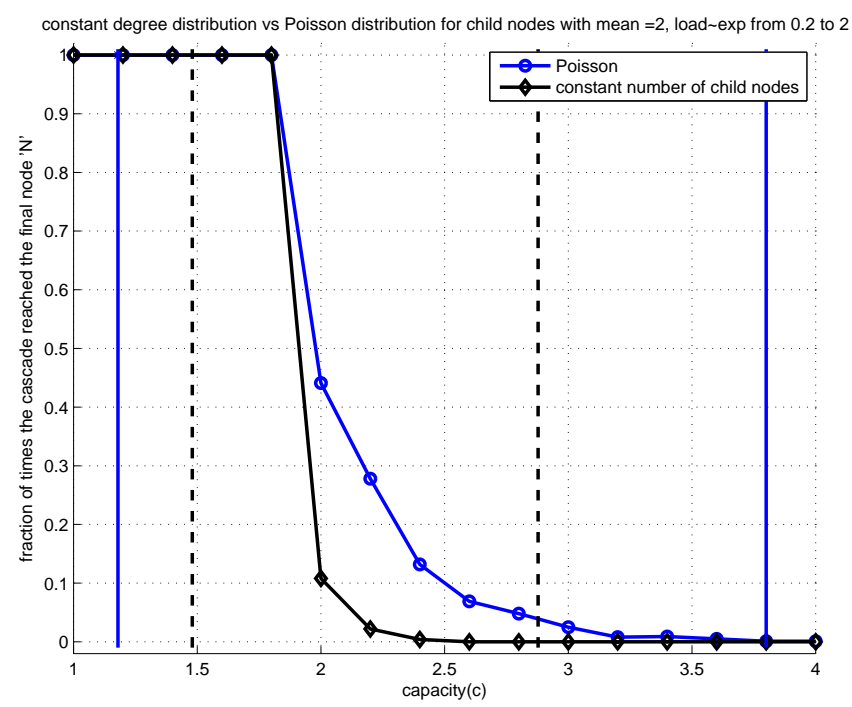

FIG. 2. Comparison of cascade probability with fixed and random tree with degree 2.

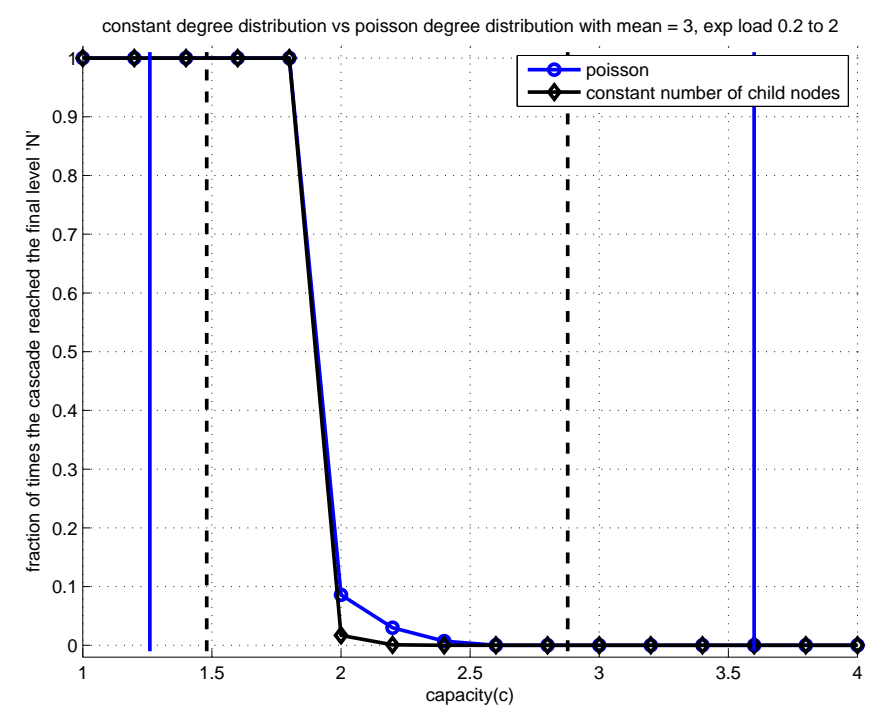

FIG. 3. Comparison of cascade probability with fixed and random tree with degree 3 .

[13] M. Lelarge, "Diffusion and cascading behavior in random networks," Games and Economic Behavior, vol. 75 , no. 2, pp. $752-775,2012$.

[14] P. Bak, C. Tang, and K. Wiesenfeld, "Self-organized criticality," Physical review A, vol. 38, no. 1, p. 364, 1988.

[15] S. N. Majumdar and D. Dhar, "Equivalence between the abelian sandpile model and the $\mathrm{i}_{i} \mathrm{q}_{i} / \mathrm{i}_{i}$ ? 0 limit of the potts model," Physica A: Statistical Mechanics and its Applications, vol. 185, no. 1, pp. 129-145,
1992.

[16] D. Dhar and S. Majumdar, "Abelian sandpile model on the bethe lattice," Journal of Physics A: Mathematical and General, vol. 23, no. 19, p. 4333, 1990.

[17] S. Manna and D. Giri, "Sandpile model with activity inhibition," Physical Review E, vol. 56, no. 5, p. R4914, 1997.

[18] M. Aizenman and J. Lebowitz, "Metastability effects in bootstrap percolation models," Journal of Physics A, pp. 22:L297-L301, 1989. 


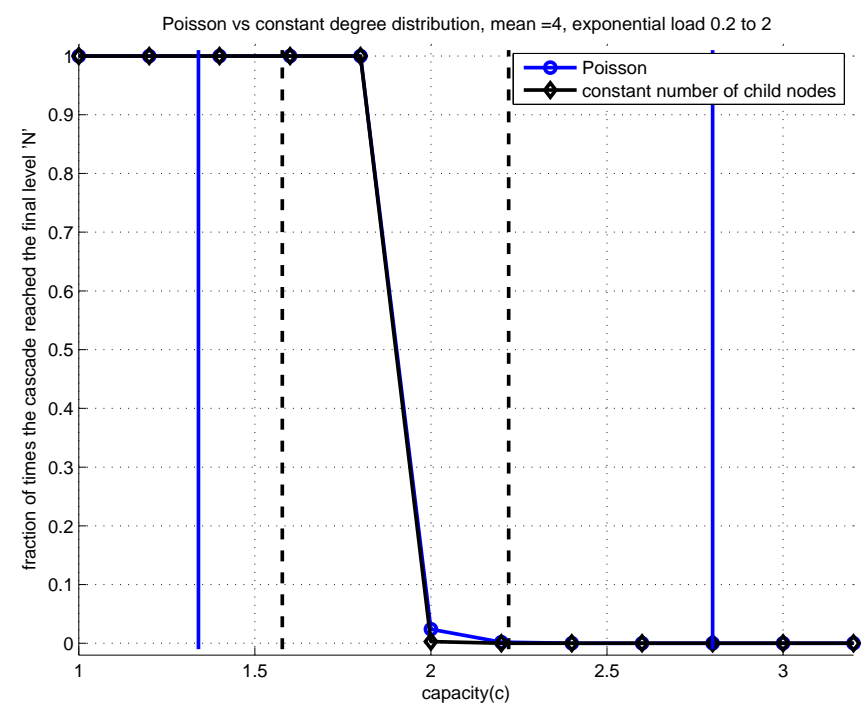

FIG. 4. Comparison of cascade probability with fixed and random tree with degree 4.

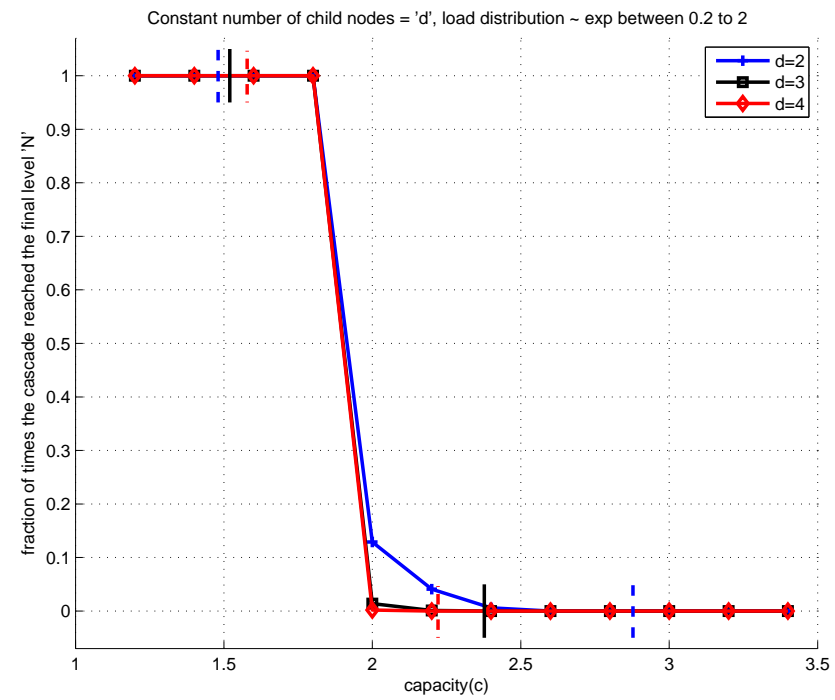

FIG. 5. Comparison of cascade probability with fixed tree for different degrees with exponential load distribution.

[19] J. Biggins, "Chernoff's theorem in the branching random walk," Journal of Applied Probability, pp. 630-636, 1977.
[20] R. Durrett, Random graph dynamics. Cambridge university press Cambridge, 2007, vol. 200, no. 7. 


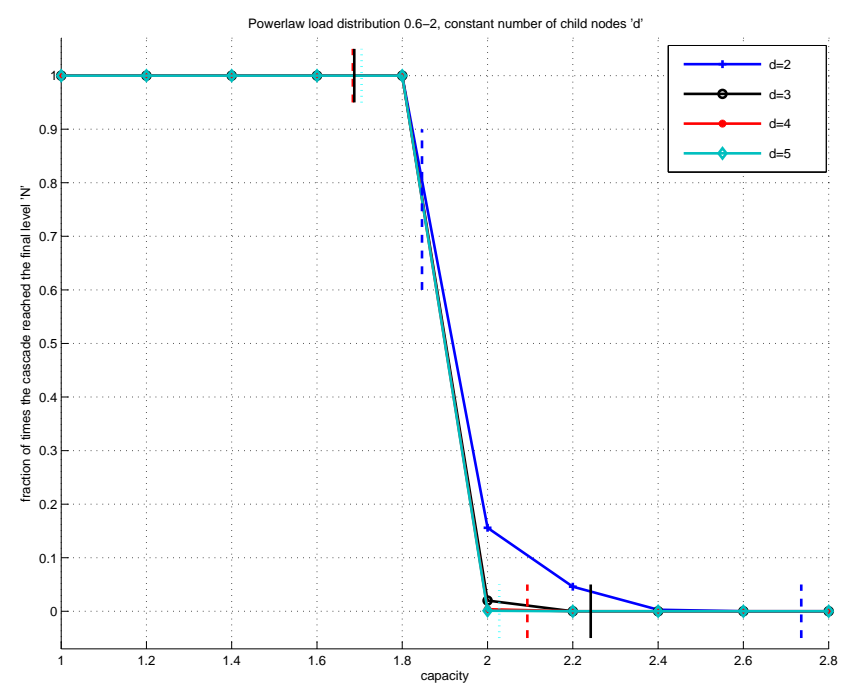

FIG. 6. Comparison of cascade probability with fixed tree for different degrees with power-law load distribution.

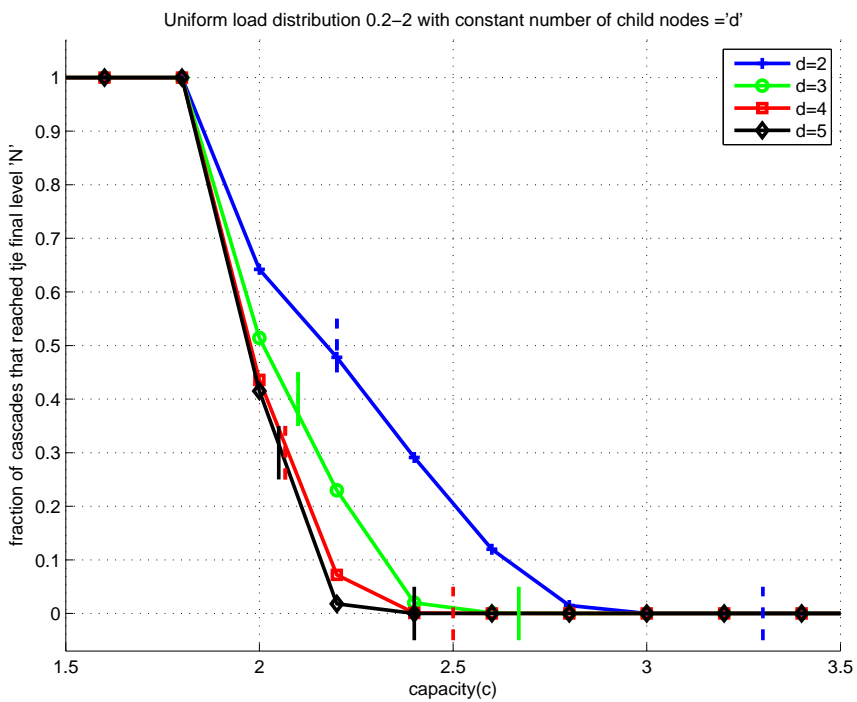

FIG. 7. Comparison of cascade probability with fixed tree for different degrees with uniform load distribution. 


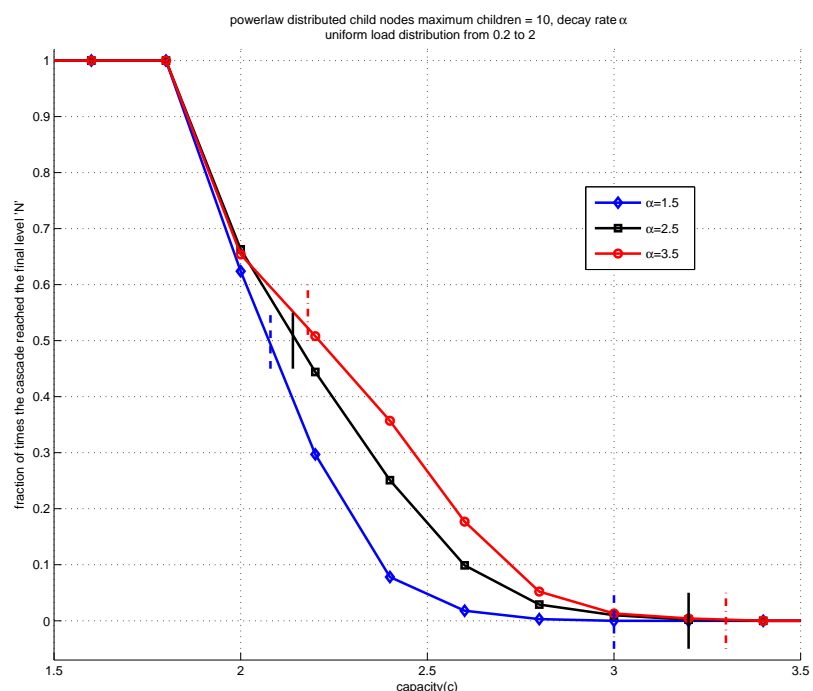

FIG. 8. Comparison of cascade probability with random tree with power-law degree distribution and uniform load distribution.

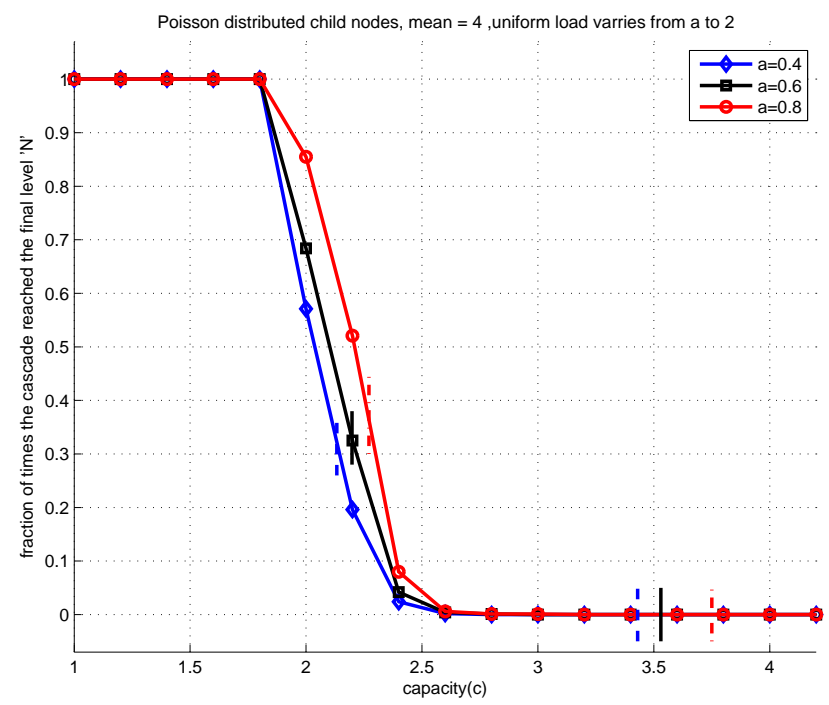

FIG. 9. Comparison of cascade probability with random tree with Poisson degree distribution and uniform load distribution. 